\title{
Ultrasound Guided Radial Arterial Catheters is More Convincing Than Blind Method
}

\begin{abstract}
:
Objective: To evaluate the effectiveness of ultrasound (US) guided radial artery cannulation as compared to the blind insertion of arterial line in intensive care unit of a tertiary care center. Methodology: One hundred (100) patients were divided into two equal groups. In group I, arterial line was inserted using the blind palpation technique. While in group II, arterial line was inserted with the help of ultrasound guidance. The primary endpoints were time of insertion in $1^{\text {st }}$ attempts, no. of first successful attempts and maximum number of attempts used for insertion of arterial line. Data was analyzed by using SPSS V23. Chi-square test was used for analysis of gender and successful insertion in $1^{\text {st }}$ attempt. Independent sample t-test and Mann-Whitney U-test were used to compare quantitative variables. Results: Mean baseline systolic blood pressure, mean diastolic blood pressure and mean pulse rate before surgery were also not significantly different between the groups. Arterial line was inserted in first attempt in in $88.0 \%$ patients in ultrasound guided group and in only $70.0 \%$ patients in blind palpation group (p-value 0.027). Arterial line insertion time in $1^{\text {st }}$ attempt was also significantly less in ultrasound guided group 77.68+7.98 seconds versus $95.46 \pm 15.53$ seconds in blind palpation group (pvalue $<0.001)$. We also found less number of insertions $1.16 \pm 0.37$ in ultrasound guided group versus $1.44 \pm 0.67$ in blind palpation group ( $p$-value 0.025 ). Conclusion: Ultrasound guided radial artery cannulation is associated with higher rate of successful insertion and less time is required for arterial line insertion as compared to blind palpation method.
\end{abstract}

Dr Nasira Pervaiz ${ }^{1}$, Dr Maria Mehmood ${ }^{2}$, Dr Shazma Ansar Butt ${ }^{3}$

1,2. Graduation, Nawaz Shareef Medical College, Gujrat

3. Grduation, Rawal Pndi Medical University, Rawalpindi

Corresponding Author:pervarisa@gmail.com

Keywords: Radial artery cannulation, Arterial line, ultrasound guided arterial line placement

DOI: $10.7176 / \mathrm{JMPB} / 62-09$

Publication date: December $31^{\text {st }} 2019$

\section{INTRODUCTION:}

Arterial line is inserted in many hospital departments e.g. operating rooms, intensive care units and emergency departments in patients in whom there is a need for continuous invasive blood pressure monitoring and arterial blood gas analysis.(1) The most frequency used site for arterial cannulation is redial artery because of it superficial location, presence of collateral blood flow through the ulnar artery and hence lower rate of complications.

Radial artery cannulation is usually performed blindly by using anatomic knowledge and palpating the artery. By using blind technique, arterial pulsation is difficultly felt in hypotensive and obese patients that can result in cannulation failure.(2) After failure of first attempt, artery may develop spam making further cannulation attempts more challenging.(3) Recent literature have suggested that ultrasound guided radial artery cannulation increases the rate of successful cannulation and decreases the rate of complications associated with arterial cannulation as compared to the traditional blind method.(4-6) The objective of this present study is to evaluate the effectiveness of ultrasound (US) guided radial artery cannulation as compared to the blind insertion of arterial line in intensive care unit of a tertiary care center.

\section{METHODOLOGY:}

Randomized prospective study design was selected for this study. Institutional review board permission was taken prior before starting the study. One hundred (100) patients were divided into two equal groups. In group I, arterial line was inserted using the blind palpation technique. While in group II, arterial line was inserted with the help of ultrasound guidance. Traditional informed consent was taken from group I patients and a special informed consent was taken from group B patients, $1^{\text {st }}$ briefing them about the efficacy of ultrasound guided atrial line insertion. The duration of this study was December 2015 to July 2016.

All patients of age more than 20 years were included in this study. Main indications of arterial line catheterization was continuous monitoring of arterial blood pressure and need for frequent arterial blood gases 
(ABGs) analysis. Hemodynamically unstable patients in whom arterial line was inserted before taking informed consent, and patients with previous attempts of radial line insertion were excluded.

Radial Artery Cannulation Technique: In all patients B-Braun Arteriofix 20G 80mm arterial line was used for radial artery access. Allen test was performed in every patient before insertion of radial line. Radial artery area disinfection and local anesthesia was given in every patient before insertion of radial line.

In group I patients, radial line was inserted using blind palpation technique according to the hospital protocols. After palpating the radial artery, the needle was inserted through the skin at 450 angel towards the anterior wall of the artery. Successful artery puncture was accessed through flashback of blood. Then a guidewire was inserted through the needle into the arterial lumen. After that needle was removed and arterial catheter was advanced over the guidewire into the arterial lumen and guidewire was removed after catheter insertion and catheter was secured into the artery.

Ultrasound guided radial line insertion was done using Toshiba Nemio 20 ultrasound machine with $7.5 \mathrm{MHz}$ linear array transducer. A sterile cover and gel was used for ultrasound transducer. Image display settings of ultrasound machine was adjusted at minimum depth of two $\mathrm{cm}$. the ultrasound probe was placed perpendicular to the artery. The artery was aligned on the centerline guide of the probe by moving the probe in exact position. The puncture needle was inserted in the skin following the centerline on the display at angel of $45^{\circ}$. After the needle punctured the arterial wall and pulsatile flow was seen in the needle, the guidewire was inserted into the artery and needle was removed. After that arterial catheter was advanced on the guidewire into the artery lumen, guidewire was removed and catheter was secured.

\section{Definitions used:}

In the ultrasound group time zero was defined as time after the machine is turned on but after application of gel on the transducer and covering it with a sterile cover. For blind palpation technique, time zero was defined as the doctor's figure was placed on the patient's wrist to palpate the radial artery. The end time of arterial line placement was noted when the catheter was successfully placed into the vessel and total time taken for insertion was calculated using a stopwatch and represented in seconds. A maximum number of three attempts for arterial puncture were decided to be used on same radial artery if failed then radial artery of the opposite hand was used for arterial line insertion.

The primary endpoints were time of insertion in $1^{\text {st }}$ attempts, no. of first successful attempts and maximum number of attempts used for insertion of arterial line.

Data was analyzed by using SPSS V23. Chi-square test was used for analysis of gender and successful insertion in $1^{\text {st }}$ attempt. Independent sample t-test and Mann-Whitney U-test were used to compare quantitative variables.

\section{RESULTS:}

One hundred patients were included in this study. There was no significant difference between age and gender of patients. Mean baseline systolic blood pressure, mean diastolic blood pressure and mean pulse rate before surgery were also not significantly different between the groups. Out of hundred patients, $46(92.0 \%)$ patients were intubated in ultrasound guided arterial line insertion group and $42(84.0 \%)$ patients were intubated in blind palpation technique group ( $\mathrm{p}$-value 0.22 ). The number of patients in which main indication for arterial line insertion was continuous arterial blood gas analysis and continuous blood pressure monitoring were same between the two groups (Table 1).

There was significant difference regarding primary endpoints between the groups. Arterial line was inserted in first attempt in in $88.0 \%$ patients in ultrasound guided group and in only $70.0 \%$ patients in blind palpation group (p-value 0.027 ). Arterial line insertion time in $1^{\text {st }}$ attempt was also significantly less in ultrasound guided group $77.68 \pm 7.98$ seconds versus $95.46 \pm 15.53$ seconds in blind palpation group (p-value $<0.001$ ) (Table 2 ). 
Journal of Medicine, Physiology and Biophysics

ISSN 2422-8427 An International Peer-reviewed Journal

Table 1. Comparison of Baseline Characteristics

\begin{tabular}{|l|l|l|l|}
\hline Variable & $\begin{array}{l}\text { Ultrasound } \\
\text { group (n=50) }\end{array}$ & $\begin{array}{l}\text { Blind Palpation } \\
\text { Group (n=50) }\end{array}$ & P-value \\
\hline Age of Patients (Y) & $44.60 \pm 7.54$ & $45.54 \pm 5.15$ & 0.45 \\
\hline Male Gender (\%) & $45(90.0)$ & $46(92.0)$ & 0.72 \\
\hline Mean Systolic Blood Pressure & $105.96 \pm 12.99$ & $108.98 \pm 14.00$ & 0.27 \\
\hline Mean Diastolic Blood Pressure & $71.60 \pm 9.28$ & $74.84 \pm 8.90$ & 0.08 \\
\hline Pulse rate per min & $76.52 \pm 7.22$ & $77.80 \pm 6.40$ & 0.35 \\
\hline Reasons of Arterial Line Insertion & \multicolumn{2}{|l|}{} \\
\hline No. of patients Intubated (\%) & $46(92.0)$ & $42(84.0)$ & 0.22 \\
\hline Continuous Arterial Blood Gas Analysis (\%) & $43(86.0)$ & $41(82.0 \%)$ & 0.58 \\
\hline Continuous Blood Pressure Monitoring (\%) & $30(60.0)$ & $32(64.0)$ & 0.68 \\
\hline
\end{tabular}

Table 2. Comparison of Study Endpoints.

\begin{tabular}{|c|c|c|c|}
\hline Variable & $\begin{array}{l}\text { Ultrasound group } \\
(\mathrm{n}=\mathbf{5 0})\end{array}$ & $\begin{array}{l}\text { Blind Palpation } \\
\text { Group }(n=50)\end{array}$ & P-value \\
\hline No. of insertions in $1^{\text {st }}$ attempt $(\%)$ & $44(88.0)$ & $35(70.0)$ & 0.027 \\
\hline No. of attempts & $1.16+0.37$ & $1.44+0.67$ & 0.025 \\
\hline Time of insertion in $1^{\text {st }}$ attempt & $77.68+7.98$ & $95.46 \pm 15.53$ & $<0.001$ \\
\hline
\end{tabular}

\section{DISCUSSION:}

The primary endpoints of this study were time of insertion in $1^{\text {st }}$ attempts, no. of first successful attempts and maximum number of attempts used for insertion of arterial line. We found significantly higher rate of arterial line access in first attempt in ultrasound guided arterial line insertion. Time of insertion was also significantly less ultrasound guided group.

Many studies have concluded that ultrasound guided arterial line insertion into the radial artery successfully increases the rate of insertion and decreases the incidence of complications as compared to the traditional blind palpation method. $(1,7,8)$ Ultrasound guided insertion is also superior in obese patients, elder patients and in patients suffering from edema, hypotension, vascular anomalies e.g. patients with tortuous vessels.(9) Sites et al. demonstrated that the most common problem of ultrasound guided arterial line insertion procedure is to accurately image the catheter during insertion.(10) Hansen et al. found that problem of image the needle can be sort out by using dynamic needle tip positioning via continuous visualization of the cannula tip and tracking the tip into the vessel lumen.(11) Previous studies have found rate of successful insertion in first attempt in 62.0 to $95.0 \% .(1,8,11-13)$ In our study, the rate of successful radial line insertion in first attempt in ultrasound guided group was $88.0 \%$ and $70.0 \%$ in blind palpation technique group.

Another beneficial use of ultrasound guided radial line insertion is to identify the patients with high risk of complications i.e. it can identify the blockage in radial or ulnar artery, and arteriosclerosis. Arteriosclerosis can be identified easily through ultrasonography,(14) so catheterization can be done in more proximal part of the radial artery. Other anatomical variations and anomalies e.g. anomalous branching of radial artery, tortuosity, radio-ulnar loop and radial artery hypoplasia can also be identified using ultrasonography. These complications are encountered in more than $17.0 \%$ patients.(15)

Radial artery cannulation using ultrasonography also have higher success rate of insertion in first attempt in pediatric patients up to $67 \%$ as compared to only $20 \%$ using blind palpation technique.

In this study, we also found less time of insertion of radial line in first attempt using ultrasound guided technique as compared blind insertion technique. We also found less number of insertions $1.16 \pm 0.37$ in ultrasound guided group versus $1.44+0.67$ in blind palpation group.

\section{CONCLUSION:}

Ultrasound guided radial artery cannulation is associated with higher rate of successful insertion and less time is required for arterial line insertion as compared to blind palpation method. 


\section{REFERENCES:}

1. Nasreen A, Khuwaja A, Akhtar P, Amjad N, Rao Z. A randomized comparison of ultrasound guided versus direct palpation method of radial artery cannulation techniques in adult patients undergoing open heart surgery. Anaesth Pain \& Intensive Care. 2016;20(1):38-42.

2. Minami T, Eisen LA, Berger JS, Sekiguchi H, Mayo PH, Narasimhan M. Gender disparity in radial and femoral arterial size: an ultrasound study. Intensive care medicine. 2007;33(3):552-3.

3. Scheer BV, Perel A, Pfeiffer UJ. Clinical review: complications and risk factors of peripheral arterial catheters used for haemodynamic monitoring in anaesthesia and intensive care medicine. Critical Care. 2002;6(3):1.

4. White L, Halpin A, Turner M, Wallace L. Ultrasound-guided radial artery cannulation in adult and paediatric populations: a systematic review and meta-analysis. British journal of anaesthesia. 2016;116(5):610-7.

5. $\mathrm{Gu}$ W-J, Wu X-D, Wang F, Ma Z-L, Gu X-P. Ultrasound guidance facilitates radial artery catheterization: a meta-analysis with trial sequential analysis of randomized controlled trials. CHEST Journal. 2016;149(1):166-79.

6. Tang L, Wang F, Li Y, Zhao L, Xi H, Guo Z, et al. Ultrasound guidance for radial artery catheterization: an updated meta-analysis of randomized controlled trials. PloS one. 2014;9(11):e111527.

7. Sandhu NS, Patel B. Use of ultrasonography as a rescue technique for failed radial artery cannulation. Journal of clinical anesthesia. 2006;18(2):138-41.

8. Shiver S, Blaivas M, Lyon M. A Prospective Comparison of Ultrasound-guided and Blindly Placed Radial Arterial Catheters. Academic emergency medicine. 2006;13(12):1275-9.

9. Shiloh AL, Savel RH, Paulin LM, Eisen LA. Ultrasound-guided catheterization of the radial artery: a systematic review and meta-analysis of randomized controlled trials. CHEST Journal. 2011;139(3):524-9.

10. Sites BD, Gallagher JD, Cravero J, Lundberg J, Blike G. The learning curve associated with a simulated ultrasound-guided interventional task by inexperienced anesthesia residents. Regional anesthesia and pain medicine. 2004;29(6):544-8.

11. Hansen M, JUHL-OLSEN P, Thorn S, Frederiksen C, Sloth E. Ultrasonography-guided radial artery catheterization is superior compared with the traditional palpation technique. Acta Anaesthesiologica Scandinavica. 2014;58(4):446-52.

12. Levin PD, Sheinin O, Gozal Y. Use of ultrasound guidance in the insertion of radial artery catheters. Critical care medicine. 2003;31(2):481-4.

13. Schwemmer U, Arzet H, Trautner H, Rauch S, Roewer N, Greim C-A. Ultrasound-guided arterial cannulation in infants improves success rate. European journal of anaesthesiology. 2006;23(06):476-80.

14. Weiner MM, Geldard P, Mittnacht AJ. Ultrasound-guided vascular access: a comprehensive review. Journal of cardiothoracic and vascular anesthesia. 2013;27(2):345-60.

15. Sarji R, Sricharoen N. Beyond the bend: a literature review and case report of radial artery loop. J Invasive Cardiol 2011; 23: E271-2. 